\title{
Informationsfluss zwischen Lethe und Mnemosyne. Zum Recht auf Vergessen, Recht auf Erinnern
}

Matthias Rossi

A. Einführung
I. Anlass: »Recht auf Vergessenwerden«

Jüngst wird eine Reform des Datenschutzes auf Ebene der EU diskutiert. Die Europäische Kommission hat im Januar 2012 den Entwurf einer EU-Datenschutzverordnung vorgestellt, ' die die derzeitige Datenschutzrichtlinie 95/46/EG ablōsen soll. Neben dem formalen Wechsel der Rechtsform von einer Richtlinie auf eine Verordnung mit all ihren Konsequenzen ${ }^{2}$ steht inhaltlich u.a. ein sog. »Recht auf Vergessenwerden« im Mittelpunkt des Entwurfs. Freilich spricht nur die Begründung des Verordnungsentwurfs davon, dass »jede Person ein Recht auf Vergessenwerden besitzen sollte, ${ }^{3}$ während der konkret anwendbare Art. 17 sodann zwar noch unter der Überschrift »Recht auf Vergessenwerden« steht, der Sache nach aber nur Löschungsgebote und Verbreitungsverbote statuiert. Die Verordnung ist in diesem Punkt also sehr viel weniger innovativ, als die Verwendung der neuen Begrifflichkeit suggerieren mag. Sie kann kein »Vergessenwerden « und deshalb auch kein »Recht auf Vergessenwerden « garantieren. Immerhin tritt aber das "Vergessenwerden« als eine maßgebliche Zielsetzung der Verordnung in den Vordergrund.

Eine solche Zielsetzung lag auch dem Wettbewerb "Vergessen im Internet" zu Grunde, der im vergangenen Jahr vom Bundesministerium des Innern gemeinsam mit der Deutschen Akademie der Technikwissenschaften veranstaltet wurde. ${ }^{4}$ Der Wettbewerb wie auch der jüngste Vorstoß im Datenschutzrecht reagieren (auch begrifflich) auf die Erkenntnis, die in der thesenartigen Formel "Das Internet vergisst nicht!« zum Allgemeinplatz geworden ist. Dieser Befund wiederum knüpft an die "Technik « des Internet an, an die nahezu grenzenlos zur Verfügung stehenden Speicherkapazitäten sowie wie vor allem an die dezentrale

1 Vorschlag der Kommission für die Datenschutz-Grundverordnung, KOM (2012), 11 v. 25.01.2012.

2 Nicht nur die nationalen Gesetzgeber, sondern auch die nationalen Verfassungsgerichte werden bei einer Verordnung weitgehend ihres Einflusses beraubt.

$3 \mathrm{KOM}$ (2012), 11, Anm. 53.

4 https://www.vergessen-im-internet.de. 
Struktur des Netzes. Die nicht erkennbare und deshalb auch nicht kontrollierbare Speicherung von Daten auf mehreren und z.T. unterschiedlichen Datenträgern führt ebenso wie die Möglichkeit der Verknüpfung von Daten dazu, dass mit der Bereitstellung von Informationen der Verlust über ihre Kontrolle, der Verlust damit auch über ihren semantischen Inhalt verbunden ist - ein Effekt, der sich durch das sog. cloud-computing jüngst noch verstärkt hat. ${ }^{5}$ Nicht zuletzt deshalb hat der Satz "Das Internet vergisst nicht! « einen deutlichen Appell-Charakter: Er versteht sich nicht nur als Befund, sondern jedenfalls auch als Warnung, insbesondere die sozialen Netzwerke und auch die Suchmaschinen nur mit Augenmaß zu nutzen. Jedenfalls will der Satz das Bewusstsein dafür fördern, dass man sich im Internet ebenso wie in der realen Welt nicht unbeobachtet bewegt, sondem Spuren hinterlässt - Spuren, die im Unterschied zur realen Welt durch die Vielzahl der Nutzer und die Perpetuierung der Daten leichter auffindbar bzw. nachvollziehbar sind. ${ }^{6}$ Deshalb schützt auch die Informationsflut, mit der sich mancher Nutzer trösten mag, allenfalls vor einer längerfristigen Aufmerksamkeit, nicht hingegen vor der grundsätzlichen Abruf- oder Rekonstruierbarkeit von Informationen.

\section{Individuelle Erinnerung und kulturelles Gedächtnis}

Die Kehrseite des Vergessens, das Erinnern, muss freilich nicht per se schädlich oder negativ beurteilt werden. Wenn Erinnern und Vergessen - jedenfalls bei anthropozentrischer Perspektive - streng in dem Sinne an eine Person gebunden sind, dass das menschliche Gedächtnis der zentrale Speicherort für Informationen ist, wird deutlich, welche Gedanken, welches Wissen Tag für Tag mit dem Tode eines Menschen erlischt: Was nicht weitergegeben wird, geht unwiederbringlich verloren. Nur wenn Informationen geteilt werden, besteht zumindest die Möglichkeit, dass sie gewärtig, dass sie gegenwärtig bleiben. Freilich mag sich dabei ihr Sinn, mag sich ihre semantische Bedeutung durch die Verknüpfung mit anderem Vorwissen, mit anderen Informationen verändern. Auch die begriffliche Unterscheidung von Daten und Informationen, ${ }^{7}$ von Tatsachen und Meinungen, von subjektiver und objektiver Auslegung, um nur einige Beispiele

5 Vgl. hierzu bspw. Wagner/Blaufuß, BB 2012, 751 ff.; Jacobs/Nägele. ZUM 2010, 281 ff.

6 Einer jüngeren Studie zufolge vergisst das Internet entgegen der allgemeinen Überzeugung doch recht schnell. wie am Beispicl von Links in Tweets zu sechs GroBereignnissen der Jahre 2009 - 2012 aufgezeigt wird: Mehr als ein Viertel der verlinkten Quellen war nicht mehr abrufbar. Vgl. hierzu Lischka, hitp:/www.spiegel.de/netzwelt/web/webarchiv-studie-zur-haltbarkeit-von-online-quellen-a-856936.html, abgerufen am 28.01.13. Präzise Masing, VVDStRL 63 (2003), 400. 
aus der juristischen Sprache zu nennen. ${ }^{\gamma}$ kann den Umstand nicht fassen, dass der Inhalt von Informationen kontextabhängig ist.

Dies gilt nicht nur für persönliches Erinnern, sondern auch für das kollektive Gedächtnis. ${ }^{9}$ Die Betrachtung des Vergangenen kann nur ex-post erfolgen und ist also mit zusätzlichen Informationen behaftet. Der Versuch, solch weiteres Erfahrungswissen durch eine vermeintlich strenge ex-ante Perspektive auszublenden, ist wohl weitgehend zum Scheitern verurteilt und weicht deshalb jüngst auch der unverblümten Suche nach neuen "Narrativen«. Doch soll es hier nicht um Geschichtsschreibung und Deutungshoheiten gehen, sondern um das Vergessen und Erinnern. Mit Blick auf das kollektive Gedächtnis einer Gesellschaft sei insofern bezweifelt, dass mehr und größere Speicher zu einer Verbesserung führen. Die Qualität des »cultural heritage« bestimmt sich ganz sicher nicht nach der Menge der verfügbaren Informationen. Auf der anderen Seite gilt für die Erinnerung einer Gesellschaft aber auch nicht der Befund, der in Bezug auf das individuelle Gedächtnis bereits für den Übergang von der mündlichen zur schriftlichen Überlieferung beobachtet wurde: "Die Kunst zu schreiben hat das Gedächtnis zu Grunde gerichtet. ${ }^{10}{ }^{0}$ Die Richtigkeit dieses Satzes mag jeder anhand seiner eigenen Mnemotechnik überprüfen. Doch in Bezug auf das kollektive Gedächtnis kann nicht geleugnet werden, dass die schriftliche Überlieferung, dass das zunächst handschriftliche, später maschinelle und nunmehr elektronische Vervielfalltigen von Büchern und Dokumenten nicht nur der Verbreitung im Raum, sondern auch der Verbreitung in der Zeit dient: Informationen werden dem Vergessen entzogen. In jedem Fall sei ins Bewusstsein gerückt, dass sich ein Recht auf Vergessen ebenso wie ein Recht auf Erinnern nicht nur subjektiv-individuell, sondern auch kollektiv-kulturell spiegeln lässt.

\section{Relevante Rechtsregeln}

Vor diesem Hintergrund soll das Recht im Folgenden nach Regelungen durchsucht werden, die das Vergessen einerseits und das Erinnern andererseits betreffen. Dabei wird es nicht bzw. nur in Ansätzen um rechtliche Regeln zur Informa-

$8 \mathrm{Zu}$ mathematischen, physikalischen und kommunikationswissenschaftlichen Begriffen der Information vgl. Gleick. Die Information: Geschichte, Begriff, Flut. 2011, passim.

9 Sehr lesenswert Dreier/Euler (Hrsg.), Kulturelles Gedächtnis im 21. Jahrhundert, 2005.

10 Kant, zitiert nach Weinrich, Lethe. Kunst und Kritik des Vergessens, 2005, S. 99, unter Bezugnahme auf folgenden Passus von Platon, Phaidros oder vom Schönen, übersetzt von Kurt Hildebrandt, 1982, S. 59: "Denn wer dies [das Schreiben] lernt, dem pflanzt es durch Vernachlässigung des Gedächtnisses Vergeßlichkeit in die Seele. weil er im Vertrauen auf die Schrift von außen her durch fremde Zeichen, nicht von innen her aus sich selbst die Erinnerung schöpft. Nicht also für das Gedächtnis, sondern für das Erinnern erfandest du ein Mittel.« 
tionsgenese gehen. Zwar hält das Recht, halten insb. die Prozessordnungen zahlreiche Regelungen bereit, die auf die Gewinnung von Informationen zielen ${ }^{11}$ oder umgekehrt bestimmte Arten der Informationserzeugung verbieten, wie etwa die Folter ${ }^{12}$ oder die nach $\S 136 \mathrm{a}$ StPO verbotenen Verhaltensweisen. ${ }^{13}$ Auch die Bestimmungen zum zivilprozessualen Strengbeweis ${ }^{1 / 4}$ und ihre Abgrenzungen zum Freibeweis ${ }^{13}$ lassen sich bei abstrakter Betrachtung als Regeln zur Gewinnung von Informationen verstehen. Und schon die Differenzierung zwischen dem verwaltungs- und strafprozessualen Untersuchungsgrundsatz und dem zivilprozessualen Beibringungsgrundsatz verdeutlicht, ${ }^{16}$ dass und mit welcher Folge das Recht Bestimmungen zur Informationsgenese enthält.

Doch in den Blick genommen werden soll das Recht hier nur in Bezug auf den Umgang mit einmal gewonnenen Informationen, auch wenn die Abgrenzung zwischen der Gewinnung von und dem Umgang mit Informationen schon wegen des Kontextbezugs von Informationen nicht gelingen kann - bei nahezu jeder

II S. die allgemeinen Rechtsgrundlagen für Datenerhebung und Informationsgewinnung im Strafverfahren $\S \S 160-163$ StPO oder $\S 406$ e StPO (Akteneinsicht im Strafverfahren); s. zur Erhebung von Informationen im Zivilprozess z.B. \$273 ZPO (Urkunden, Auskünfte), $\$ \$ 287$ Abs. 1 S. 3, 448, 452 Abs. 1 ZPO (Vernehmung); $\$ 395$ Abs. 2 ZPO (Angaben zur Person in der Beweisaufnahme); im Verwaltungsverfahren z.B. $\$ \S 87,87 \mathrm{~b}, 99$ VwGO (Beziehen von Akten, Urkunden und Auskünften), § 100 Abs. 1 VwGO (Akteneinsicht der Beteiligten); vgl. zum Ganzen auch Werner, in: Roßnagel (Hg.), Handbuch Datenschutzrecht, 2003, 8.1 Rn. 11 ff., 8.2 Rn. 7 ff., Rn. 111 ff.

$12 \S 343 \mathrm{StGB}$ stellt die Erpressung von Aussagen durch Amtsträger unter Strafe, Art. 104 Abs. 1 S. 2 GG untersagt Folter festgehaltener Personen; ein absolutes Folterverbot statuieren Art. 4 EU-GRCh, Art. 3 EMRK.

$13 \S 136$ a StPO verbietet die Beeinträchtigung der Willensfreiheit durch »Misshandlung, durch Ermüdung, durch körperlichen Eingriff, durch Verabreichung von Mitteln, durch Quålerei, durch Täuschung oder durch Hypnose " (Abs. 1) sowie durch "Maßnahmen, die das Erinnerungsvermögen oder die Einsichtsfahigkeit des Beschuldigten beeinträchtigen« (Abs. 2). § 136a Abs. 3 StPO statuiert diesbezüglich ein Verwertungsverbot.

14 Beweis wird nur nach dem gesetzlich vorgeschriebenen Verfahren mit den in $\$ \S 355 \mathrm{ff}$. ZPO genannten Beweismitteln geführt, § 284 ZPO, s. Prütting, in: Münch-Komm, § 284 ZPO Rn. 26.

15 Das Gericht entscheidet über Verfahren und Beweismittel, der Freibeweis erfordert gem. $\S 284$ S. 2 ZPO die Zustimmung beider Prozessparteien und ist grundsätzlich nicht widerruflich, s. Prütting (Fn. 14). \$284 ZPO Rn. 26.

16 Zum verwaltungsprozessualen Untersuchungsgrundsatz. der eine amtswegige Untersuchung und Klärung des Sachverhalts erfordert. Kopp/Schenke, VwGO, 18. Aufl. 2012, $\$ 86 \mathrm{Rn} .1 \mathrm{ff}$. und Sodan/Ziekow, VwGO, 3. Aufl. 2010, § 86 Rn. 2 ff. Zum Untersuchungsgrundsatz für die Staatsanwaltschaft und das Gericht im Strafprozess MererGoßner, StPO, 55. Aufl. 2012, Einl. Rn. 10 mit Verweis auf $\$ \$ 160,244$ Abs. $2 ; \S 155$ Rn. 12 sowie Becker, in: Löwe-Rosenberg. Die Strafprozessordnung und das Gerichtsverfassungsgesetz, 26. Aufl. 2010, $\$ 244 \mathrm{Rn}$. $39 \mathrm{ff}$. Demgegenüber besagt der zivilprozessuale Beibringungsgrundsatz (Verhandlungsgrundsatz) kurzum, dass der Streitstoff ausschließlich von den Parteien beigebracht werden kann, siehe etwa Thomas/Putzo, ZPO, 33. Aufl. 2012, Einl. I, Rn. 1 ff., Vorbem. \$253 Rn. 35 ff.; vgl. auch Baumbach/u. a., ZPO, 71. Aufl. 2013, Grdz. $\S 128$ Rn. 20 f. 
Verwendung von vorhandenen Informationen werden neue generiert. Gleichwohl beschränkt sich der Beitrag auf die Suche nach rechtlichen Regelungen über den Umgang mit solchen Informationen, die vergessen oder erinnert werden sollen.

\section{Lethe und Mnemosyne}

Hier nun erklärt sich auch der Verweis auf die griechische Mythologie, auf die beiden Flüsse in der Unterwelt, Lethe und Mnemosyne, die vereinfacht gesprochen für das Vergessen und das Erinnern stehen. Das Vergessen, bewirkt durch das Trinken vom Wasser der Lethe, wird dabei als notwendiges Ritual verstanden, um sich nach der Wiedergeburt nicht an vorherige Leben zu erinnern. Ähnlich wie die Worte Vergils beschreiben: "Die Seelen, die, göttlicher Weisung fügsam, erneut sich verkörpern, trinken vom Wasser der Lethe, schlürfen mit ihm Vergessenheit ein und Freiheit von Sorgen. "17, stellt die Ausübung eines Rechts auf Vergessen ein neues Leben in Aussicht, frei von Erinnerungen an ein altes, sei es ein reales, sei es ein virtuelles. Mancher Internetnutzer wird seine Informationen insofern gerne der Lethe anvertrauen, während manche "Datenkrake« wie Apple, Microsoft, google, Facebook u.a. sicherlich lieber aus der Mnemosyne trinken wollen. Denn wer von ihrem Wasser trinkt, der wird sich an alles erinnern und in der Folge allwissend sein.

Anders als die Kräfte der Lethe und der Mnemosyne kann das Recht den Informationsfluss in letzter Konsequenz nicht bestimmen. Aber es enthält erstaunlich viele Regelungen, die das Vergessen und Erinnern begünstigen.

\section{B. Grundrechtliche Verwurzelungen}

Die Grundrechte verhalten sich zu einem Recht auf Vergessen und einem Recht auf Erinnern - jedenfalls in ihrer Gesamtschau - neutral. Denn den Grundrechten, denen sich Ansprüche auf ein Vergessen entnehmen lassen, stehen Grundrechte gegenüber, die für eine Erinnerung streiten.

Ausgangspunkt der individual-freiheitlich ausgerichteten Grundrechteordnung ist dabei das Selbstbestimmungsrecht des Einzelnen. Es manifestiert sich im Allgemeinen Persönlichkeitsrecht $t^{18}$ und wird konkretisiert durch das Recht auf informationelle Selbstbestimmung. ${ }^{19}$ Doch so sehr das Allgemeine Persönlichkeits-

17 Vergil, Werke in einem Band, herausgegeben und aus dem Lateinischen übersetzt von Dietrich Ebener, 2. Aufl. 1987, S. 290.

18 Grundlegend BVerfGE 35, 202.

19 Grundlegend BVerfGE 65, 1. 
recht auch dem Schutz des "Rufes" einer Person zu dienen bestimmt ist, so wenig gibt es dem Einzelnen ein ausschließliches Verfügungsrecht über die Darstellung der eigenen Person. Seine einzelnen Ausprägungen, insbesondere etwa das Recht am gesprochenen bzw. geschriebenen Wort, das Recht am eigenen Bild oder auch das Recht auf Wissen um die eigene Abstammung, ${ }^{20}$ lassen sich zwar als Recht verstehen, selbst zu bestimmen, was über die eigene Person erinnert und was vergessen werden soll. Doch das Bundesverfassungsgericht hat früh herausgestellt, dass »der Einzelne [...] eine sich innerhalb der sozialen Gemeinschaft entfaltende Persönlichkeit [ist], $\varkappa^{21}$ dass der Mensch also ein »soziales Wesen « ist, dessen Freiheit der Selbstbestimmung und -entfaltung »als die eines gemeinschaftsbezogenen und gemeinschaftsgebundenen Individuums zu verstehen ist. ${ }^{22}$ Die Freiheit, das Bild von der eigenen Person durch die Hervorhebung bestimmter und die Negierung anderer Informationen prägen zu können, stößt insoweit auf Schranken, die ihrerseits grundrechtlich fundiert sind. Namentlich die Meinungsfreiheit und allen voran auch die Pressefreiheit können dem individuellen Wunsch nach Vergessen ein kollektives Interesse an Erinnerung entgegen- und sich damit häufig auch durchsetzen, wie etwa die Judikatur zu den Persönlichkeitsrechten Prominenter im Spannungsverhältnis zum Interesse der Öffentlichkeit an $»$ Personen der Zeitgeschichte ${ }^{23}$ oder auch die Entscheidungen in der Sache "Spickmich.de ${ }^{24}$ zeigen.

Gleiches gilt für das Recht auf informationelle Selbstbestimmung, das das Bundesverfassungsgericht als eigenes Grundrecht aus dem (ebenfalls richterrechtlich begründeten) Allgemeinen Persönlichkeitsrecht abgeleitet hat. Die Herrschaft über die eigenen Daten, die den Kerninhalt des Rechts ausmachen, ist nicht unbeschränkt, da der Mensch ein auf Kommunikation angewiesenes Sozialwesen ist. ${ }^{25}$ Gleichwohl ist das Recht, ngrundsätzlich selbst über die Preisgabe und Verwendung seiner persönlichen Daten zu bestimmen, « von entscheidender Bedeutung für das Vergessen und Erinnern personenbezogener Informationen, denn es schützt »gegen unbegrenzte Erhebung, Speicherung, Verwendung und Weitergabe ${ }^{26}$ persönlicher Daten.

Der Ausgleich zwischen den z.T. widerstreitenden Grundrechten wird durch den Gesetzgeber geschaffen, der im demokratischen Staat im besten Falle zu-

$20 \mathrm{Vgl}$. zu den einzelnen Rechten die Auflistung durch das Bundesverfassungsgericht selbst, BVerfGE 54, 148, 154; jüngst Bezug nehmend BVerfGE 114, 339, 346.

21 BVerfGE $33,303,334 ; 45,187,227 ; 117,71,89$.

22 BVerfGE 4, 7, 15; ständige Rechtsprechung.

23 Vgl. hierzu umfassend Lenski, Personenbezogene Massenkommunikation als verfassungsrechtliches Problem, 2007, passim.

24 BGHZ 181, 328; BVerfG, Beschl. v. 16.08.2010, vgl. hierzu Ladeur, JZ 2009, 966 ff.; Haensle/Reichold. DVBI. 2009, $1329 \mathrm{ff}$.

25 BVerfGE 65, 1, 43 f.

26 BVerfGE 65, 1, 43. 
gleich die gesellschaftliche Meinung widerspiegelt. Freilich wird etwa in Bezug auf die Frage, wann welche Vorstrafen aus den Registern gelöscht und damit vergessen werden können, eine Diskrepanz zwischen der auch rechtsstaatlich geprägten parlamentarischen Gesetzgebung und der stärker emotional beeinflussten "Volksmeinung" festzustellen sein. Auch in anderen Rechtsbereichen, etwa dem Datenschutz oder allgemein in Fragen der Verjährung von Taten oder auch nur von Ansprüchen, wird "Volkes Wille« in Abhängigkeit vom Grad der persōnlichen Betroffenheit oder öffentlichen Aufmerksamkeit von den gesetzlichen Bestimmungen abweichen. Gleichwohl ist es gerade diese in ihrem abstraktgenerellem Charakter liegende Distanz der Gesetze, die diese als Gradmesser für die Kultur des Vergessens und Erinnerns tauglich erscheinen lāsst. Deshalb seien im Folgenden einige Rechtsbereiche näher betrachtet.

C. Vergessensregelungen in ausgewählten Rechtsbereichen

I. Datenschutzrecht

Die wohl offenkundigsten Regelungen zum Vergessen finden sich im Datenschutzrecht. Grundrechtlich verwurzelt im Recht auf informationelle Selbstbestimmung, hält das Datenschutzrecht in verschiedenen Bestimmungen eine feine Abstufung des Vergessens bereit.

\section{Löschung von Daten}

Sichtbarster Ausdruck des intendierten Vergessens sind die Ansprüche auf Löschung von Daten. Als Löschen wird von $\S 3$ Abs. 4 S. 2 Nr. 5 BDSG das Unkenntlich machen gespeicherter personenbezogener Daten bezeichnet. Erfasst wird jede Handlung, die dazu führt, dass Daten nicht mehr zur Kenntnis genommen und Informationen nicht länger aus gespeicherten Daten gewonnen werden können. ${ }^{27}$ Entscheidend ist dabei, dass die Daten nicht mehr lesbar sind. ${ }^{28}$

Daten sind immer dann zu löschen, wenn eine Speicherung unzulässig ist oder eine Kenntnis für die Erfüllung des Zwecks der Speicherung überhaupt nicht mehr erforderlich ist. Eine Löschung verlangt, dass jegliche Reproduzierbarkeit ausgeschlossen ist. Die Gewährleistung ist funktional zu begreifen, so dass keine negativen Rückschlüsse etwa aus der Schwärzung der Eintragung in der Spalte

28 Gola/Schomerus, BDSG, 11. Aufl. 2012, § 3 Rn. 40. 
"Straftaten am Arbeitsplatz" möglich sind ${ }^{29}$ - das Vergessen soll effektiv bewirkt werden.

\section{Sperrung von Daten}

Ein abgeschwächtes Instrument auf dem Weg zum Vergessen ist die Sperrung. $\S 3$ Abs. 4 S. 2 Nr. 4 BDSG bezeichnet das Sperren als das Kennzeichnen gespeicherter personenbezogener Daten, um ihre weitere Verarbeitung oder Nutzung einzuschränken. Das Sperren intendiert und bewirkt ein weitgehendes Verarbeitungsverbot der gespertten Daten, das allein durch Kennzeichnung gewährleistet wird. Diese Gewährleistung ist funktional zu verstehen, so dass auch aus der Kennzeichnung als gesperrt, etwa im Datenfeld für Vorstrafen, keine negativen Rückschlüsse gezogen werden dürfen. ${ }^{30} \mathrm{Im}$ Unterschied zur Löschung kann die Sperrung allerdings in der Regel rückgängig gemacht werden. Denn die Informationen werden nicht physisch vernichtet, sondern nur in besonderer Weise gekennzeichnet und in ihrer Verfügbarkeit beschränkt. Hier zeigt sich, dass die Sperrung unmittelbar als Verwendungsverbot und nur mittelbar als Vergessensgebot wirkt, was in der Terminologie des Entwurfs der EU-DatenschutzGrundverordnung dadurch zum Ausdruck kommt, dass an Stelle des Begriffs "sperren" das "Recht auf Beschränkung der Datenverarbeitung" verwendet wird. $^{31}$

Die Sperrung kommt an Stelle der Löschung grundsätzlich in zwei Konstellationen in Betracht. Sie wird zum einen angeordnet, wenn eine Löschung entweder zu aufwändig oder gar unmöglich ist (vgl. bspw. $§ 48$ Abs. 2 S. 2 ASOG). Sie ist zum anderen aber vor allem dann das richtige Instrument, wenn sich die Richtigkeit von Informationen nicht klären lässt, ${ }^{32}$ es also im Rahmen der Beweislastverteilung zu einem »non-liquet« kommt. ${ }^{33}$

Auch ein drittes Betroffenenrecht im Datenschutz lässt sich als ein Recht auf Vergessen verstehen. Das Berichtigungsrecht gibt den Betroffenen die Möglichkeit, auf den Inhalt der gespeicherten Informationen einzugehen und somit zu verhindern, dass ein falsches Bild von seiner Person perpetuiert wird. Unrichtige Daten sollen vergessen, nur die "wahren« Angaben sollen erinnert werden. Für

29 Dammann (Fn. 27), § 3 Rn. 177; Innenministerium Baden-Württemberg, StAnz. 27/1979. 5; Senatskommission für das Personalwesen, ABl. der Freien Hansestadt Bremen 1979, 392.

30 Dammann (Fn. 27), \& 3 Rn. 168.

31 KOM (2012), 11 endg., S. 10.

32 Vgl. z.B. BVerwGE 131, 171, Rn. 41 = NVwZ 2008, 1371 (1375).

33 Mallmann, in: Simitis (Hg.). BDSG, 7. Aufl. 2011, § 20 Rn. 55; Wedde, in: Roßnagel (Hg.), Handbuch Datenschutzrecht, 2003, 4.4 Rn. 75. 
die Richtigkeit der Daten trägt der Betroffene die Beweislast. Kann der Beweis nicht geführt werden, kommt nur eine Sperrung der Daten in Betracht.

\section{Daten in Zentralregistern}

Von besonderer Bedeutung sind weiterhin Löschungsrechte hinsichtlich Daten in zentralen Registern. Denn grundsätzlich werden Informationen nur deshalb in zentralen Registern abgelegt, um sie dem Vergessen zu entziehen. Sie sollen personen-, amts- und kompetenzunabhängig erinnert werden. Doch der Erinnerungsfähigkeit des Staates sind grundrechtliche Grenzen gezogen.

\section{Bundeszentralregister}

So enthält das BZRG zahlreiche differenzierte Bestimmungen für die Eintragung und die Tilgung von Haft- und Geldstrafen, ${ }^{34}$ der Sperrung von Fahrerlaubnissen, von Behörden- und Gerichtsentscheidungen etc. Von besonderer Bedeutung sind die Einträge für Straftäter. Das Bundesverfassungsgericht hat diesbezüglich früh entschieden, dass mit Blick auf deren intendierte Resozialisierung bestimmte Informationen dadurch vergessen werden müssen, dass entsprechende Einträge im Bundeszentralregister gestrichen werden. Vor diesem Hintergrund ist es durchaus problematisch, wenn die in den staatlichen Registern festgehaltenen Informationen auch nach Ablauf ihrer "Haltbarkeit» durch private Initiativen perpetuiert werden, wie es etwa bei einer privat initiierten Karte über Wohnorte von Sexualstraftätern oder auch bei der Aufrechterhaltung von Onlinearchiven der Fall ist. ${ }^{35}$ Private Pranger, die durch die "Schwarmintelligenz" des Internet relativ einfach errichtet werden können, rufen deshalb die grundrechtlichen Schutzpflichten auf den Plan, die freilich auch von den potentiellen Opfern etwaiger Wiederholungstaten in Anspruch genommen werden.

\section{Verkehrszentralregister}

Das allgemeine Persönlichkeitsrecht, das bei dem Gedanken der Resozialisierung in besonderer Weise berührt ist, ist unzweifelhaft weniger beeinträchtigt bei Ein-

$34 \S 32$ BZRG regelt die Nichtaufnahme bestimmter Verurteilungen in das Führungszeugnis; die Tilgung von Eintragungen bestimmt sich nach $\$ 45$ BZRG, die Entfernung nach $\S 24$ BZRG; s. Tolzmann, in: Roßnagel (Hg.), Handbuch Datenschutzrecht, 2003, 8.6 Rn. $101 \mathrm{ff}$. zum Beschwerdeverfahren.

35 Vgl. hierzu jüngst BGHZ 183, $353 \mathrm{ff}$. 
trägen in das Verkehrszentralregister in Flensburg. Doch auch hier sind die Löschungspflichten wegen der enormen praktischen Bedeutung dieses Registers rechtlich von besonderer Bedeutung, ${ }^{36}$ denn auch unterhalb der Schwelle einer Kriminalisierung trägt die sogenannte Punktedatei in gewisser Weise zu einer Stigmatisierung bei, die noch dazu handfeste freiheitsrelevante rechtliche Konsequenzen nach sich ziehen kann. Die Tilgung der Eintragung der Punkte nach Maßgabe des § 29 StVG dokumentiert insoweit ein Recht auf Vergessen, das für jeden Verkehrsteilnehmer von alltäglicher Bedeutung ist.

\section{Zivil- und presserechtliche Bestimmungen}

Auf ein Vergessen zielen auch zahlreiche Regelungen im Privatrecht. Im Zusammenhang mit dem Schutz der persönlichen Ehre sind vor allem Unterlassungsansprüche, darüber hinaus aber auch Schadenersatzansprüche von Bedeutung.

Geprägt werden die einzelnen Instrumente von der Überlegung, dass der Ruf einer Person nur begrenzt durch die Person selbst bestimmt werden kann. Sie hat keinen Anspruch auf ein bestimmtes Bild, keinen Anspruch auf eine bestimmte

Wahrnehmung ihrer selbst durch andere und durch die Öffentlichkeit. ${ }^{37}$ Der Ausgleich zwischen dem allgemeinen Persönlichkeitsrecht und dem Presserecht. das insoweit stellvertretend steht für das öffentliche Interesse, ist grundrechtlich nur schwer zu fassen, wie die zum Teil divergierenden Entscheidungen des Bundesverfassungsgerichts auf der einen Seite und des EGMR auf der anderen Seite zeigen. $^{38}$

\section{Unterlassungsansprüche}

Die Instrumente, die das Presserecht insoweit zur Korrektur, im besten Fall gar zum Vergessen bestimmter Informationen bereithält, beginnen schon bei den bloßen Unterlassungsansprüchen. Zwar sind solche Unterlassungsansprüche noch nicht unmittelbar auf Vergessen gerichtet, mittelbar allerdings tragen sie durchaus zu einem Vergessen bei, weil durch die verbotene und deshalb unterlassene Wiederholung einer Aussage die maßgebliche Information in Vergessenheit gerät. Dass eine Information dabei häufig erst mit der Berichterstattung über

36 Zweckbestimmung und zu speichernde Datenkategorien des Verkehrszentralregisters sind in der durch das StVG-Änderungsgesetz (BGBI. I 1998, 747) neu gefassten Rechtsgrundlage $\$ 28$ StVG aufgelistet.

37 BVerfGE 99, 185, 194; 101, 361, 380.

38 Vgl. EGMR. NJW 2004. 2647; BVerfGE 101, $361 \mathrm{ff}$. 
die Geltendmachung des Unterlassungsanspruchs in die Öffentlichkeit und damit in das allgemeine Bewusstsein gerät, gehört zu den Widersprüchlichkeiten, die von jedem Anspruchsinhaber stets eine sorgfältige Abwägung erfordern. Zuweilen ist es besser, eine zwar falsche, aber nicht allgemein bekannte Äußerung über sich zu dulden, als öffentlich gegen die Information vorzugehen. Die Möglichkeit, das entsprechende Gerichtsverfahren unter Ausschluss der Öffentlichkeit stattfinden zu lassen, ${ }^{39}$ ist hier in den weitaus meisten Fällen nur ein schwaches Korrektiv.

\section{Recht auf Gegendarstellung}

Auch das presserechtliche Recht auf Gegendarstellung lässt sich - wie schon der Berichtigungsanspruch im Datenschutzrecht - als ein Recht auf Vergessen verstehen. Selbst wenn es unmittelbar nicht zu einem Vergessen derjenigen Daten beiträgt, zu denen die Gegendarstellung erfolgt, so vermag es doch erstens die Richtigkeit dieser Information in Zweifel zu ziehen und zweitens Dritten eine Grundlage dafür zu bieten, sich ein eigenständiges Bild zu machen. Insoweit werden die Informationen nicht gänzlich vergessen, aber sie werden unter Umständen korrigiert. Für das allgemeine Persönlichkeitsrecht ist schon viel gewonnen, wenn für die Zukunft haften bleibt, dass eine bestimmte Äußerung inhaltlich umstritten war.

\section{Schadenersatzansprüche}

Ob schließlich auch die möglichen Schadenersatzrechte im Presserecht eine Vergessensfunktion erfüllen, mag auf den ersten Blick bezweifelt werden. Doch schon wegen ihrer potentiellen präventiven Wirkung, die in Deutschland wegen der meist geringen Höhe des Schadenersatzrechts freilich wenig ausgeprägt ist, lassen sie sich als vergessensgerichtete Ansprüche verstehen.

IV. Strafrechtliche Tatbestände zum Schutz der persönlichen Ehre

Auch die strafrechtlichen Tatbestände zum Schutz der persönlichen Ehre entfalten über ihre repressive Wirkung eine präventive Funktion. Namentlich die sogenannten Äußerungsdelikte, also etwa die Strafbarkeit der Beleidigung oder der 
üblen Nachrede, sind auch darauf angelegt, solche ehrverletzende Informationen zu vergessen.

\section{Fristen}

Noch allgemeiner lassen sich Fristen jeder Art als Vergessensregelungen verstehen. Wenn Einwendungen nur binnen einer bestimmten Frist von Bedeutung sind, danach aber irrelevant werden, dann ist ein Vergessen der jeweiligen Informationen die Folge. Die Präklusionsvorschriften im Öffentlichen Recht mit ihrer Differenzierung zwischen der formellen und der materiellen Präklusion ${ }^{40}$ sind ein konkretisierendes Beispiel für solche Regelungen. Aber auch die allgemeinen Fristen von Rechtsmitteln tragen letztlich zu einem Vergessen bei. Sie verstehen sich als einen Ausgleich zwischen den beiden Polen der materiellen Rechtmäßigkeit auf der einen Seite und der Rechtssicherheit auf der anderen Seite und ordnen im Interesse letzterer den Ausschluss bestimmter Einwände nach den gesetzlich fixierten Fristen an.

\section{Verjährung}

Rechtssicherheit und Rechtsfrieden sind letztlich auch Ziele von Verjährungen. ${ }^{41}$ Als rechtliches Instrument des "Vergessens « lassen sich deshalb auch die zahlreichen Verjährungsvorschriften begreifen, die das gesamte Recht mit all seinen Teildisziplinen erfassen. Bei streng juristischer Betrachtung ordnen sie kein Vergessen an, weil sie nur als Strafverfolgungshindernis wirken bzw. die Durchsetzbarkeit eines weiterhin bestehenden Anspruchs oder Rechts hindern. ${ }^{42}$ Gleichwohl bewirken sie im Ergebnis - und dies ist auch eine ihrer Intentionen - dass der Fall vergessen wird. Sie zielen insofern auf Rechtssicherheit und Rechtsfrieden zugleich. ${ }^{43}$

40 Siehe hierzu Schmidt-Aßmann, in: Maunz/Dürig, GG-Kommentar, 2012, Art. 19 Rn. $258 \mathrm{ff}$.

41 Zu den zahlreichen Funktionen von Verjährungen vgl. Guckelberger, Die Verjährung im Öffentlichen Recht, 2004, 72 ff.

42 BGHSt 1I, 394; Peters/Jacoby, in: Staudinger, BGB, Neub. 2009, Vorbem. \$§ 194-225 Rn. 4.

43 Begründung zum Regierungsentwurf zum Schuldrechtsmodernisierungsgesetz. BTDrucks. $14 / 6040$ S. 100. 
Als Vergessensvorschriften in großer Form lassen sich schließlich Amnestien verstehen, die das Vergessen bereits begrifflich zum Ausdruck bringen. ${ }^{44}$ Hier wird das Vergessen quasi von Staats wegen verordnet. Während für Individuen der Erlass von Steueramnestien in der heutigen Zeit von vorrangiger Bedeutung ist, ${ }^{45}$ können Amnestien grundsätzlich auch auf andere Delikte bezogen sein. Das Vergessen ist dabei zwar nicht immer das eigentliche Ziel, in jedem Fall aber die Wirkung solcher allgemeinen Strafentlassungen, die durch so banale Gründe wie die beschränkte Kapazität von Gefängnisplätzen oder mangelnde "wirtschaftliche Effizienz« begründet sein mögen.

Auch Friedensverträge, die unter anderem begangene Kriegsverbrechen für nicht verfolgbar erklärten, hatten eine enorme Bedeutung für das kollektive Gedächtnis. ${ }^{46}$ In der rechtlich und vor allem menschenrechtlich zunehmend durchwobenen Welt wird heute freilich häufig ein anderer Weg gegangen, um die Vergangenheit zu bewältigen: Wahrheitskommissionen sollen gerade nicht ein Vergessen, sondern umgekehrt eine Aufbereitung und somit eine Erinnerung sicherstellen. ${ }^{47}$

\section{Vergessensverbote}

Auf der anderen Seite gibt es auch regelrechte Vergessensverbote. Zu nennen ist sicherlich zunächst die Ausnahme von Verjährungen. Mord verjährt nach $\S 78$ Abs. 2 StGB ebenso wenig ${ }^{48}$ wie Völkermord, Verbrechen gegen die Menschlichkeit und Kriegsverbrechen nach $\S 5$ des Völkerstrafgesetzbuchs: Sie sind der Strafverfolgung und damit auch der Erinnerung niemals entzogen. Auch dem $\mathrm{Zi}$ vilrecht sind unverjährbare Rechte nicht fremd. Das betrifft insbesondere die

44 Das altgriechische wamnstia« wird mit "Vergessen. Vergeben « übersetzt.

45 Vgl. hierzu Gehm, ZRP 2010, $169 \mathrm{ff}$.

46 In diesem Zusammenhang mag erinnert werden, dass Churchill in seiner Züricher Rede. die zwar natürlich nicht als Friedensvertrag zu qualifizieren ist, die aber den Weg von verfeindeten Nationalstaaten zu »Vereinigten Staaten von Europa« aufzeigt. den früheren englischen Premierminister Gladstone zitiert: „a blessed act of oblivion « - den segensreichen Akt des Vergessens.

47 Bspw. die Truth and Reconciliation Commission in Sūdafrika 1996; Kommission für Wahrheit und Versöhnung in Jugoslawien 2003.

48 Zur Unverjährbarkeit bei »Mord« im Strafrecht Mitsch, in: Münch-Komm StGB. 2. Aufl. 2012, § $78 \mathrm{Rn} .14 \mathrm{f}$. 
Persönlichkeitsrechte sowie die dinglichen Rechte, namentlich also das Eigentum. $^{49}$

\section{Recht auf Erinnern}

Dies leitet über zu einem Recht, vielleicht gar einer Pflicht zur Erinnerung. Um noch einmal an die EU-Datenschutzgrundverordnung anzuknüpfen, sei insoweit darauf hingewiesen, dass entgegen dem »Recht auf Vergessenwerden « die weitere Speicherung der Daten zulässig sein soll, wenn dies für historische oder statistische Zwecke, zum Zwecke der wissenschaftlichen Forschung, aus Gründen des öffentlichen Interesses im Bereich der öffentlichen Gesundheit oder zur Ausübung des Rechts auf freie Meinungsäußerung erforderlich ist. ${ }^{50}$

\section{Gedenktage}

Sichtbarster Ausdruck einer kollektiven Erinnerung sind darüber hinaus die staatlichen Gedenktage und die gesetzlichen Feiertage. Erinnert sei an den 27. Januar als Tag des Gedenkens an die Opfer des Nationalsozialismus, der auf den Tag der Befreiung des Konzentrationslagers Auschwitz gelegt wurde. ${ }^{51}$ Der 8. Mai wird als Tag der Befreiung gefeiert, wobei bereits in der Bezeichnung eine bestimmte Wertung mitschwingt, die bereits eine (erfreuliche) Distanz zur bedingungslosen Kapitulation der Wehrmacht erkennen lässt. Bereits vor der Wiedervereinigung war der 17. Juni als nationaler Gedenktag in der Bundesrepublik an den Aufstand des 17. Juni 1953 in der DDR anerkannt. Am 20. Juli wird an das fehlgeschlagene Attentat auf Adolf Hitler erinnert, das maßgeblich mit dem Namen Stauffenberg verbunden ist. Und der 9. November schließlich hat für Deutschland eine Reihe von verschiedenen Bedeutungen - er erinnert an die Novemberrevolution 1918 mit der Ausrufung der Deutschen Republik durch Philipp Scheidemann, an den euphemistisch als Reichskristalinacht bezeichneten Beginn der Novemberpogrome 1938 sowie an die Öffnung der deutsch-deutschen Grenze, an den Mauerfall im Jahre 1989. Der zweite Sonntag vor dem ersten Advent wird als Volkstrauertag staatlich als stiller Tag geschützt, an dem die Erinnerung

49 Siehe zu unverjährbaren familienrechtlichen Ansprüchen und einer Auflistung übriger unverjährbarer zivilrechtlicher Ansprüche, Grothe, in: Münch-Komm BGB, 6. Aufl. $2012, \$ 194$ Rn. 7 ff., \$ 195 Rn. 37.

50 KOM (2012), 11 endg.. S. 29; Vorschlag für die Datenschutz-Grundverordnung. Erwägungsgrund 53.

51 Vgl. hierzu das Gelöbnis von Elie Wiesel in der Erzählung "Nuit«: "Jamais je l'oublierais.« 
an die Kriegstoten und Opfer der Gewaltherrschaft aller Nationen wachgehalten werden soll.

Die exakte rechtliche Fassung all dieser Gedenk- und Feiertage mit ihrer Differenzierung zwischen staatlich anerkannten Feiertagen, kirchlichen Feiertagen, geschützten Tagen und stillen Tagen, die in ihrer konkreten Ausgestaltung noch dazu entsprechend der bundesstaatlichen Untergliederung in die Kompetenzen der Länder fällt, spielt in diesem Kontext keine Rolle. ${ }^{52}$ Entscheidend ist, dass der Staat an bestimmten Tagen die Erinnerung an historische Ereignisse, zum Teil natürlich auch an religiöse Ereignisse, festgehalten wissen will.

\section{Denkmalschutz als Ausprägung des Schutzes des kulturellen Erbes}

Eine ähnliche Intention verfolgt auch das Denkmalschutzrecht, das sich als Ausprägung des Schutzes des kulturellen Erbes ebenso als Status quo Sicherung zur Erinnerung begreifen lässt. Unabhängig davon, ob das Denkmalschutzrecht auf Bau- und Bodendenkmale, auf Immobilien und bewegliche Sachen oder auf Gesamtanlagen gerichtet ist, stets geht es um die Erinnerung, geht es also darum, Informationen der Lethe zu entreißen und sie mit Mnemosyne für die Zukunft festzuhalten, sie zu vererben. Das Übereinkommen zum Schutz des Kultur- und Naturerbes der Welt, das 1972 von der UNESCO beschlossen wurde und 1975 in Kraft trat, verdeutlicht die weltweite Bedeutung solcher Erinnerungen dabei ebenso sehr wie die grundsätzlich anthropozentrische Ausrichtung des Kulturund Denkmalschutzes. ${ }^{53}$

\section{Archivwesen}

Die gleiche Zielsetzung verfolgen die zahlreichen Bibliotheken, Museen und Archive, die neuerdings als sogenannte Gedächtnisinstitutionen bezeichnet wer$\operatorname{den}^{34}$ und bereits hier auf das maßgebliche Ziel der Erinnerung hinweisen. Die jeweiligen Archivierungsvorgaben, ebenso aber auch die allgemeine Zugäng-

52 Vgl. hierzu bspw. Christian Hufen, Der Ausgleich verfassungsrechtlich geschützter Interesen bei der Ausgestaltung des Sonn- und Feiertagsschutzes, 2013, passim.

53 Die Erinnerungsfunktion kommt im Übrigen in der englischen Bezeichung des UNProgramms »Memory of he World» besser zum Ausdruck als in der deutschen Bezeich-

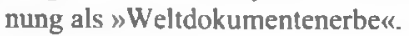

54 Vgl. etwa die Diskussion um den Entwurf zur Fortentwicklung der PSI-Richtlinie, die die Weiterverwendung aller Informationen der öffentlichen Hand und somit auch die Bestände von Museen. Bibliotheken und Archiven betrifft. Siehe hierzu die Stellungnahme des Deutschen Bibliotheksverbandes vom 6. Juli 2012. zugänglich unter www.bibliotheksverband.de. 
lichkeit solcher Informationen zeigen, dass es dem Staat hier um die Sicherung eines kollektiven Gedächtnisses geht. Im Einzelnen mögen sie dabei völlig unterschiedlich wirken. Denn mag die einzelne Information für sich genommen auch unangetastet bleiben, mag also etwa bei einem in einem Museum befindlichen Bild das Bild als solches nicht verändert und der Nachwelt überliefert werden, so besteht doch die Besonderheit in der Speicherung dieser Informationen darin, dass diese Informationen in ihrem Zusammenhang, gegebenenfalls aber auch mit Wissen von außen, neu interpretiert und auf diese Art und Weise zu einer neuen Informationsgenerierung beitragen können. Obwohl als Informationen verstanden, werden im Ergebnis somit eher Daten gespeichert und einer neuen inhaltlichen Anreicherung offengehalten.

\section{Mnemopolitik}

Vor dem Hintergrund der sachlichen Vielfalt und vor allem auch der kompetenziellen Diversifizierung ließe sich fragen, ob der Staat nicht eine kohärente Mnemopolitik betreiben sollte. In der Schweiz jedenfalls hat das dortige Bundesamt für Kultur eine solche Politik initiiert bzw. jedenfalls das Bewusstsein für die Notwendigkeit einer kohärenten Speicherung und Weitergabe des kulturellen Erbes geschaffen. ${ }^{55}$

\section{E. Überflüssigkeit eines expliziten Rechts auf Vergessenwerden}

Vor dem Hintergrund dieser knappen und bei weitem nicht vollständigen Bestandsaufnahme ist der Triumph eines Rechts auf Vergessenwerden, den der Entwurf der EU-Datenschutzverordnung in der öffentlichen Diskussion verzeichnen kann, kaum nachvollziehbar. Vielmehr gibt es aus rechtlicher Sicht zahlreiche Bedenken gegen die Normierung einer entsprechenden Regelung.

\section{Missachtung des Subsidiaritätsprinzips}

Diese Bedenken knüpfen zunächst generell an das Subsidiaritätsprinzip an. ${ }^{56} \mathrm{Ne}-$ ben den Einwendungen, die sich gegen die Rechtsform einer Verordnung erheben ließen, ist speziell in Bezug auf das Recht auf Vergessenwerden fraglich, ob

55 Vgl. den Bericht des Bundesamtes für Kultur zur Mnemopolitik des Bundes vom November 2008 , zugänglich unter www.bak.ch.

56 S. auch die Subsidiaritätsrüge des Bundesrates, BR-Drs. 52/12 vom 30. März 2012. 
eine einheitliche Regelung auf Unionsebene notwendig ist. Eine solche Bestimmung ignoriert, dass bereits zivilrechiliche Instrumente bestehen, die dem Einzelnen gewähren, »von dem für die Verarbeitung Verantwortlichen die Löschung von sie betreffenden personenbezogenen Daten und die Unterlassung jeglicher weiteren Verbreitung dieser Daten zu verlangen, ${ }^{57}$ Denn abgesehen von den skizzierten Bestimmungen des Datenschutzrechts und des Presserechts, abgesehen also von den Unterlassungspflichten und Berichtigungsansprüchen bei ehrverletzenden Äußerungen im Internet gegen Betreiber von Internetforen gem. § 1004 Abs. 1 S. 2 BGB $^{58}$ analog iVm $\$ 823$ BGB oder $\S \S 4$ Abs. 1,35 Abs. 3 $\mathrm{BDSG},{ }^{59}$ sei insofern für das deutsche Recht in Erinnerung gerufen, dass Content-Provider $^{60}$ nach $\S \S 7-10$ TMG grundsätzlich verschuldensunabhängig haften, woraus sich auch Unterlassungspflichten ergeben. ${ }^{61}$ Auch Host-Provider, die anderen auf ihren Servern Speicherplatz zur Verfügung stellen, haften nach \$ 10 TMG in Abhängigkeit von ihrer Kenntnis. ${ }^{62}$ Ebenso sei hervorgehoben, dass das BDSG auch auf Server außerhalb der EU anwendbar ist, wenn die Daten in Deutschland abgerufen werđen. ${ }^{63}$ Dass all diese Rechte nicht ausreichen, um den Einzelnen vor einer von ihm nicht gewollten und von der Rechtsordnung nicht tolerierten Perpetuierung seiner Informationen zu schützen, so dass dieses Zie] »besser « auf Unionsebene erreicht werden kann, ist nicht ersichtlich.

\section{Legistische Alternativen}

Doch nicht nur die bestehenden Regelungen lassen Zweifel an der Notwendigkeit einer unionsweiten Bestimmung aufkommen. Vielmehr lassen sich auch legistische Alternativen zu einem hoheitlich verankerten subjektiven Recht auf Vergessenwerden denken. In Betracht kommen vor allem zivilrechtliche bzw. marktwirtschaftliche Lösungen, die den Wunsch nach Vergessenwerden möglicherweise besser gerecht werden könten als die Eintäumung eines subjektiven Anspruchs. Längst hat der Markt die Nachfrage nach dem Löschungsbedürfnis erkannt und durch entsprechende Angebote reagiert. »Online-Reputationsmanager $(\rightsquigarrow \mathrm{ORM} \ll)^{64}$ oder weputation defender ${ }^{65}$ nennen sich die neuen

57 So Art. 17 Abs. 1 Vorschlag zur Datenschutz-Grundverorỏnung. KOM (2012), 11 endg. S. 58 .

58 Z.B. BGH, Urt. v. 27. März 2007, VI ZR 10/106,

59 Gola/Schomerus (Fr. 28), \& 35 Rn. 2b.

60 Stellt eigene Inhalte zur Verfügung.

61 Muller-Broich, TMG, \& 7 Rn. 10.

$62 \mathrm{Vgl}$. zur Haftungsfrage der verschiedenen Provider Sieber/Höfinger, in: Hoeren/Sieber (Hrsg.), Handbuch Multimedia-Recht, 32. EL 2012. Anm. 18.1.

63. BGH, MMR 2012, 124

64 Vgl, für Untermehmen, Ziegelmaver, GRUR 2012, $761 \mathrm{ff}$. 
Dienstleister, die unter den Web-Adressen "web-killer.de» oder »ndeinguterruf.de « versprechen, unliebsame Inhalte im Internet zu löschen. ${ }^{66}$ Als eigene Dienstleistung ist dabei eine Datensuche vorgeschaltet, die nach bestimmten Begriffen oder Fotos sucht und so den Gegenstand des Vergessens überhaupt erst ausmacht.

\section{Technische Realisierbarkeit}

Freilich sei eingeräumt, dass marktwirtschaftliche wie hoheitliche Lösungen unter ein und derselben Schwierigkeit stehen. $\mathrm{Ob}$ nämlich der versprochene und damit geschuldete Erfolg tatsächlich bewirkt werden kann, mag angesichts des technischen Phänomens Internet schon aus technischen Gründen fraglich sein. Gelöscht werden die Informationen aus einem Speicher. Doch ob sie aus allen Speicherm gelöscht werden, vermag niemand zu garantieren. Der besondere Charakter von Informationen, der darin liegt, dass sie nicht ausschließlich und ausschließend sind, sondern trotz Teilung vollständig beim Inhaber verbleiben, steht einer Kontrolle über alle Speicher entgegen. Insbesondere kann nicht ausgeschlossen werden, dass die Informationen auch bei ihrer physischen Vernichtung in den Köpfen vorhanden bleiben. Und eine Rekonstruierbarkeit von Informationen ist durch die zahlreichen Möglichkeiten einer Rekombination nicht vorhersehbar. Im Ergebnis muss man sich darüber bewusst sein, dass das Recht ein Löschen zwar bestimmen, das Vergessen aber nicht erzwingen kann.

\section{F. Verwertungsverbote statt Vergessensrechte}

Das Recht wäre gut beraten, wenn es sich auf solche Folgen beschränken würde. die es auch gewährleisten kann. Deshalb spricht viel dafür, an Stelle von Vergessensrechten Verwertungsverbote zu normieren. Der Sache nach lassen sich Löschungspflichten und ebenso wie Rechte auf Löschen bzw. Vergessenwerden als gesteigerte Form von Verwertungsverboten, nämlich als absolute Verwertungsverbote begreifen.

Freilich ist die Rechtsordnung in Bezug auf Verwertungsverbote nicht kohärent. Sie bewegt sich vielmehr am Rande ihrer Glaubwürdigkeit, wenn sie zum Teil aus verbotenem Wissen weitgehende rechtliche Konsequenzen ableitet. Die

65 Weichert, RDV 2007, 54 ff.; ders., DuD 2009, 7 ff.; Gola/Schomerus (Fn. 28). $\$ 35$ Rn. 2a.

66 Schimansky, Weiße Weste im Web? Vergessen Sie's!, Welt Online vom 4. Juni 2012. verfügbar unter: http://www.welt.de/106411476. 
unter Folter erlangten Informationen gelten nach Völkerrech ${ }^{67}$ und auch nach nationalem Recht nichts, ${ }^{68}$ der heimliche Tonbandmitschnitt hilft im Zivilrecht nicht weiter, ${ }^{69}$ doch ausgerechnet im Strafrecht ${ }^{70}$ - und im Steuerrecht ${ }^{71}$ - sollen rechtswidrig erlangte Informationen nicht einfach vergessen werden, sondern auf den Prüfstand einer Abwägung gestellt werden, die im Ergebnis meist zu Gunsten des Erinnems ausfällt.

Unabhängig davon, ob und mit welchem genauen Gehalt ein »Recht auf Vergessenwerden« deshalb Eingang in die Rechtsordnung findet, wird beim Schutz der eigenen Persönlichkeit künftig wohl verstärkt Eigenverantwortung und das heißt "Datenvermeidung « im Vordergrund stehen. Denn der vielzitierte Satz mag zwar einige Plausibilität, angesichts der Effizienz von Suchmaschinen woh! aber keine Gültigkeit mehr für sich in Anspruch nehmen können und somit letztlich nur Ausdruck einer unbegründeten Hoffnung bleiben: »Gespeichert, das heißt vergessen."

67 Vgl. Art. 5 AEMR: Art. 7 IPbpR; Art. 3 EMRK sowie vor allem die UNAntifolterkonvention.

68 Vgl. zum Fall Daschner LG Frankfurt. Urt. v. 20.12.2004 - Az. 5/27 KLs 7570 Js $203814 / 03$ (4/04), 5-27 KLs 7570 Js $203814 / 03(4 / 04)=$ NJW 2005. 692; s. dazu Braum. KritV 2005, 282 ff. einerseits und Götz, NJW 2005, 953 andererseits.

69 Zum Verwertungsverbot von heimlichen Tonbandaufnahmen als Beweismittel im Zivilprozess s. BGH, Urt. v. 18.2.2003 - Az. XI ZR 165/02 = NJW 2003, 1727; Foerste, in: Musielak (Hrsg.), ZPO Kommentar, 9. Aufl. 2012, \$284 Rn. 23.

70 Zur Diskussion um die wfruits of the poisoned trees vgl. Eisenberg. StPO Spezialkommentar, 7. Aufl. 2011, Rn. 404 ff.; s. auch BGHSt 34, 362: BVerfGK 16. 22. das die Verwertbarkeit von Beweismitteln, die bei einer rechtswidrigen Wohnraumdurchsuchung gewonnen worklen sind, bejaht.

71 Zur Verwertung illegal erworbener Informationen uber Steuerhinterzieher s. BVerfGK 18, 193, das die Verwertbarkeit von Beweisen, die von Informanten unter strafbewehrter Weise zur Verfügung gestellt wurden bejaht; zur Problematik insgesamt s. Kaiser, NStZ 2011, 383. 